\title{
Finite element analysis of stress distribution and the effects of geometry in a laser-generated single-stage ceramic tile grout seal using ANSYS
}

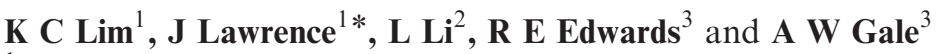 \\ ${ }^{1}$ Manufacturing Engineering Division, School of Mechanical and Production Engineering, Nanyang Technological \\ University, Singapore \\ ${ }^{2}$ Laser Processing Research Centre, Department of Mechanical, Aerospace and Manufacturing Engineering, \\ University of Manchester Institute of Science and Technology, Manchester, UK \\ ${ }^{3}$ Department of Civil and Construction Engineering, University of Manchester Institute of Science and Technology, \\ Manchester, UK
}

\begin{abstract}
Optimization of the geometry (curvature of the vitrified enamel layer) of a laser-generated single-stage ceramic tile grout seal has been carried out using a finite element (FE) model. The overall load-bearing capacities and load-displacement plots of three selected geometries were determined experimentally by the indentation technique. Simultaneously, an FE model was developed utilizing the commercial ANSYS package to simulate the indentation. Although the loaddisplacement plots generated by the FE model consistently displayed stiffer identities than the experimentally obtained results, there was reasonably close agreement between the two sets of results. Stress distribution profiles of the three FE models at failure loads were analysed and correlated so as to draw an implication on the prediction of a catastrophic failure through an analysis of FE-generated stress distribution profiles. It was observed that although increased curvatures of the vitrified enamel layer do enhance the overall load-bearing capacity of the singlestage ceramic tile grout seal and bring about a lower nominal stress, there is a higher build-up in stress concentration at the apex that would inevitably reduce the load-bearing capacity of the enamel glaze. Consequently, the optimum geometry of the vitrified enamel layer was determined to be flat.
\end{abstract}

Keywords: optimization, curvature, grout, enamel, glaze, load-bearing capacity, finite element (FE), ANSYS, stress distribution

\section{INTRODUCTION}

Vitrified tile is one of the most preferred floor surfaces due to its durability, high chemical resistance and ease of cleaning. Generally, vitrified tiles are secured to a concrete substrate with tile grout or adhesive. To account for inherent dimensional inaccuracies, adjoining tiles are purposely separated by a void, which is then filled with a commercially available grout. Since most grouts are epoxy based, they have several inherent problems. The critical problem with epoxy-based grouts is their inability

\footnotetext{
The MS was received on 8 August 2003 and was accepted after revision for publication on 25 June 2004.

* Corresponding author: Manufacturing Engineering Division, School of Mechanical and Production Engineering, Nanyang Technological University, 50 Nanyang Avenue, Singapore 639798. E-mail: mjlawrence@ ntu.edu.sg
}

to restrict the movement of microparticles and microorganisms; contaminants, germs and bacteria can enter and exit the grout into cavities behind the tiles to corrode the adhesive between the tile and substrate [1]. Moreover, hygiene of the environment cannot be controlled. Consequently, epoxy-based grouts cannot be applied in places that demand strict compliance with cleanliness regulations, such as clean rooms and surgical operating theatres. Also, epoxy-based grouts do not have a strong resistance against wear. Under routine cleaning of the tiled surface with water and cleaning agents, these epoxy-based grouts wear out very quickly. In addition, cleaning of these grouts requires much effort and time. Therefore, the useful life of the epoxy-based grout is greatly reduced. Finally, epoxy-based grout seals become contaminated and damaged over time and, in turn, have to be removed physically and mechanically, which is arduous and costly. 
The inherent problems with commercial epoxy-based grouts motivated Lawrence and co-workers [2-5] to develop the single-stage ceramic tile grout sealing process. In this approach, a single pass of a high-power diode laser beam (HPDL) creates a vitrified platform that wets and bonds a layer of enamel frit to a substrate of crushed vitrified ceramic tiles and simultaneously seals off the surface with an enamel glaze. The mechanical, chemical and physical characteristics of the single-stage ceramic tile grout seal have been shown to be superior to those of a conventional epoxy-based grout [6]. In particular, the rupture strength of the enamel glaze on single-stage ceramic tile grout was found to be similar to that of the vitrified glaze of a ceramic tile. Although the single-stage grout seal has a strong vitreous enamel glaze, the concave surface attained upon firing is vulnerable to intensive compressive loads. At the initial loading, the enamel glaze is first to fail. As loading progresses, stress build-ups in the vitrified tile substrate will cause the single-stage grout seal to collapse. It is therefore relevant to study the effects of the curvature of the vitrified enamel layer on the stress distribution in a single-stage ceramic tile grout seal and optimize the geometry for compressive loads. Due to the dimensional smallness of the seal, the depth-sensing indentation technique was employed to determine its mechanical properties.

Previously, from an analysis of stress distribution Zhang et al. [7] and Bull et al. [8] have shown that the geometry of a weld bead plays an important role in determining the mechanical properties of the weld. Teng et al. [9] demonstrated experimentally that the fatigue life in butt-welded joints can be improved by modifying the weld geometry and residual stresses. In addition, Karkhin [10] explained that the efficiency and stress distribution of the weld is related to its weld shape. By employing the finite element (FE) method, Shuzalec [11] demonstrated that the mechanical performance and stress concentration in a weld joint can be improved and minimized through shape optimization. Moreover, there is no significant loss of accuracy in simulating indentation testing with the FE method, as an analysis by Dong and Darvell [12] showed that the results yielded by an FE model were in reasonable agreement with actual indentation results.

In this work, an analysis of the stress distribution in a laser-generated single-stage ceramic tile grout seal under compressive indentation is to be carried out with the FE approach using the commercial simulation package, ANSYS 6.0. The ultimate aim of this analysis is to optimize the geometry of the upper curved surface of the single-stage ceramic tile grout seal in terms of failure loads, which will give an indication of the catastrophic failure load and the overall load-bearing capacity.

\section{EXPERIMENTAL PROCEDURES}

\subsection{Sample preparation}

For experimental purposes, standard $150 \mathrm{~mm} \times$ $150 \mathrm{~mm} \times 5 \mathrm{~mm}$ vitrified ceramic tiles were cut into smaller pieces of $20 \mathrm{~mm} \times 20 \mathrm{~mm} \times 5 \mathrm{~mm}$ and applied in pairs to an ordinary Portland cement (OPC) substrate using standard epoxy tile grout. The width of the void was $2 \mathrm{~mm}$. The fixed ceramic tiles were then allowed to set for a standard setting period of $24 \mathrm{~h}$. Vitrified ceramic tiles were crushed, fine-ground by a pestle and mortar and sieved to ensure a particle size that was smaller than $30 \mu \mathrm{m}$. To form a manageable paste the vitrified ceramic powder was mixed with $50 \mathrm{wt} \%$ water-diluted sodium silicate solution and was applied into the void to a height of $4 \mathrm{~mm}$ and allowed to cure for $8 \mathrm{~h}$. Simultaneously, another manageable paste was formed by mixing enamel frits with $20 \mathrm{wt} \%$ white spirit. A thin enamel frit paste layer with a thickness of $0.5 \mathrm{~mm}$ was overlaid on the cured vitrified ceramic tile compound and allowed to cure for $1 \mathrm{~h}$. Finally, the single-stage grout-sealing process was completed by irradiating the compound and sealing the substrate with enamel glaze with a single pass of the HPDL. All the three test geometries had a basic depth of $4.5 \mathrm{~mm}$, as shown in Fig. 1 . Specific curvatures of the vitrified enamel layer were achieved by adjusting the distribution and quantity of the enamel frits and by varying the laser processing parameters.

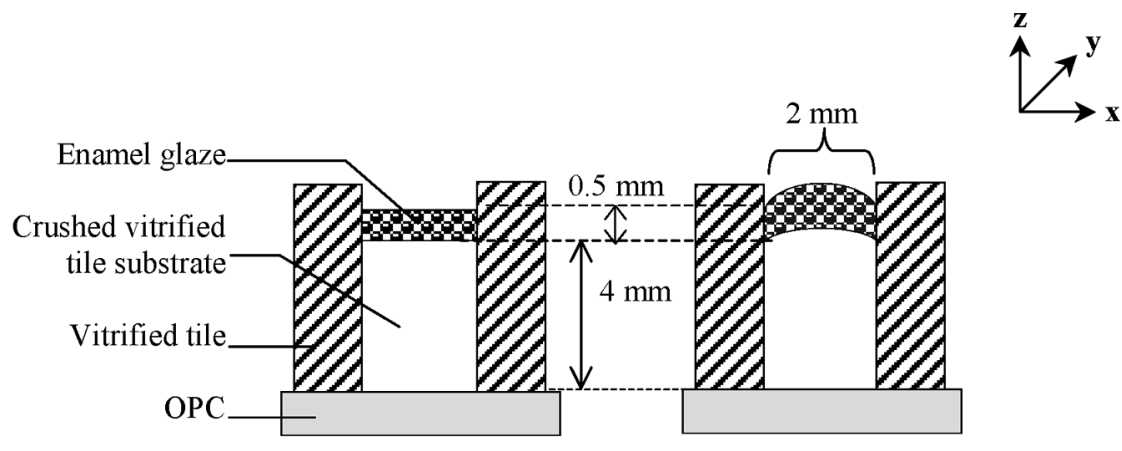

Fig. 1 Schematic cross-sectional view of the single-stage ceramic tile grout seal samples 


\subsection{Indentation testing procedure}

Indentation testing was carried out by the Instron Universal Tester in accordance with an international standard [13]. A compressive test rig equipped with a working tool of a cone indentor with a contact face of a nominal diameter of $1 \mathrm{~mm}$ was utilized. The test samples were placed on to the work-stage of the compressive test rig. The position of the test sample was adjusted so that the centre-line of the single-stage grout seal aligned with the projected path of the indentor. During indentation, the cross head along with the indentor travelled downwards at a slow, constant velocity of $0.2 \mathrm{~mm} / \mathrm{min}$. During the travel, the applied force was ramped up to a maximum load that caused the specimen to fail. A gauge-length extensometer measured the displacement of the indentor with increasing load at a sampling rate of $2 \mathrm{pts} / \mathrm{s}$. The experimental data were fed back into a computer program which computed and outputted the displacement-load history.

\section{DEFINING THE CURVATURE OF THE VITRIFIED ENAMEL LAYER}

Most researches in two-dimensional shape optimization use a mathematical function or a methodology to define and represent a curved boundary. Pedersen and Lauren [14], Kristensen and Madsen [15] and Bhavikatti and Ramakrishnan [16] have all used the polynomial function, whereas Braibant and Fleury [17], Yang and Choi [18] and Sandgren and Wu [19] have each used a spline curve. Meanwhile, Hsu et al. [20] reasoned that there has yet to be an explicit method to define and represent a curved boundary by successfully conducting shape optimization on a torque arm with a curvature function using the FE method.

In this work, the curvature of the vitrified enamel layer is the only variable to define the differing concave and convex surface topographies. With only two distant points defined in space, the spline curve method to define the surface topography would require a great deal of guesswork and is therefore inappropriate. Although the polynomial function can define a smooth boundary, it is not such an appealing approach as it would require many tedious mathematical transformations for the different magnitudes of curvature of the vitrified enamel layer. Consequently, a new approach was adopted using the trigonometric shape function, $N$, given by

$$
N=\sin \left[\left(\frac{X+W / 2}{W}\right) \pi\right]
$$

which would ensure a smooth boundary for successful two-dimensional shape optimization and the mathematical function, $Y$, with a built-in trigonometric identity,

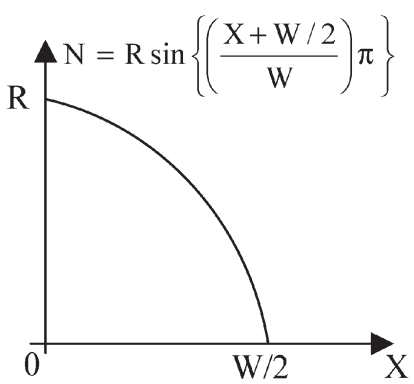

Fig. 2 Shape function for the surface topography

given by

$$
Y=R \sin \left[\left(\frac{X+W / 2}{W}\right) \pi\right]
$$

where $X$ is the distance from the centre of the single-stage ceramic tile grout seal, $W$ is the width of the single-stage ceramic tile grout seal and $R$ is the curvature of the vitrified enamel layer. Surface topography of differing curvatures of the vitrified enamel layer, regardless of whether they be concave or convex, can be defined by adjusting $R$.

It can be seen from Fig. 2 that this shape function has $N=\sin (\pi)=0$ at $X=W / 2$ and, at $X=0$, where $N=\sin (\pi / 2)=R$, the maximum or minimum surface topography displacement can be obtained by multiplying by a positive or negative curvature of the vitrified enamel layer. A concave surface has a positive $R$, whereas a convex surface has a negative $R$. The surface topography of the single-stage ceramic tile grout is thus modelled with a series of key points that reasonably define the geometry. To closely follow the surface topography, $X$ was incremented from 0 to $1 \mathrm{~mm}$ at intervals of $0.05 \mathrm{~mm}$. Seven geometries listed in Table 1 were investigated. Three of these geometries, 1, 4 and 5, were compared with experimental values obtained from actual indentation tests to allow correlation of the results with FE models.

\section{DEVELOPMENT OF THE FINITE ELEMENT MODEL}

\subsection{Element selection}

The SOLID95 element was used for the three-dimensional modelling of the single-stage ceramic tile grout

Table 1 List of geometries investigated

\begin{tabular}{lc}
\hline Geometry & Curvature $(\mathrm{mm})$ \\
\hline 1 & 0 \\
2 & +0.2 \\
3 & +0.4 \\
4 & +0.7 \\
5 & +1.2 \\
6 & -0.2 \\
7 & -0.7 \\
\hline
\end{tabular}

Proc. Instn Mech. Engrs Vol. 218 Part B: J. Engineering Manufacture 
seal. This element comprises four configurations: brick, pyramid, tetrahedral and prism. The 20-node brick element is the basic configuration that handles regularshaped blocks. Volumes of irregular shapes are handled by the other three configurations: tetrahedral (10 nodes), pyramid (13 nodes) and prism (15 nodes). The SOLID95 element is versatile as it can tolerate irregular shapes and could therefore model the curved boundaries of the single-stage ceramic tile grout seal without much loss of accuracy. Each node has three degrees of freedom, translations in the nodal $x, y$, and $z$ directions, which are sufficient to handle the deformation in the singlestage ceramic tile grout seal during indentation testing.

\subsection{Material properties}

As the HPDL beam impinges with the surface of the single-stage ceramic tile grout seal, vitrification takes place in a downwards direction from the surface. Although the top layer is homogeneous due to sufficient mixing between the enamel frit and the crushed vitrified ceramic tile, the bottom layer is non-homogeneous. Therefore, the gradual decrease in the vitrification level as the depth increases indicates that the microstructure of the lower layers is anisotropic and, consequently, its mechanical properties are spatially inconsistent. Since Fisher-Crisps [21] has shown that the elastic modulus of functionally graded material derived from current conventional methods has a discrepancy with the actual value, using such a functionally graded elastic modulus will most certainly extend the computational time but will not necessarily improve accuracy.

Although anisotropy introduces additional material properties that render the basic field equation better structured, more accurate and easier to solve, as suggested by Azhdari et al. [22], many simplified FE models assume isotropic conditions. A good example is a recent study by Dong and Darvell [12], who represented a ceramic-cement substrate under indentation by an FE model with isotropy and obtained reasonable agreement in calculated and observed values of the contact radius. Since the composition of the single-stage ceramic tile grout seal is similar to the ceramic-cement substrate materials studied by Dong and Darvell, it is reasonable to simplify and assume the single-stage ceramic tile grout seal to be homogeneous, isotropic and having a constant elastic modulus. The assumed mechanical properties of the single-stage ceramic tile grout seal are listed in Table 2. The elastic modulus

Table 2 Mechanical properties for the single-stage ceramic tile grout seal

\begin{tabular}{ll}
\hline Elastic modulus & $77.0 \mathrm{GPa}$ \\
Poisson's ratio & 0.189 \\
Compressive yield strength & $100 \mathrm{MPa}$ \\
\hline
\end{tabular}

and Poisson's ratio are close to that of glass since these parameters of the mix are determined primarily by the water-glass after firing [23]. The compressive yield strength is assumed to be close to that of a vitrified tile.

The failure analysis, an idealized Tresca criterion, is appropriate for the brittle single-stage ceramic tile grout seal. Fisher-Cripps [21] found that a mica-containing glass-ceramic material exhibits shear-driven yield in an indentation. Furthermore, it was seen that micacontaining glass-ceramic materials exhibit yield in indentation tests with spherical indentors, even at modest loads. In addition, the indentation response of these glass-ceramic materials was found to be a function of the macroscopic uniaxial compressive yield stress when the material was modelled, using the FE method, as an elastic-plastic solid in conjunction with the Tresca shear-stress failure criterion. Other workers $[\mathbf{2 4}, \mathbf{2 5}]$ have also idealized a brittle or nearly brittle material to be an elastic, perfectly plastic characteristic, governed by a Tresca failure criterion.

\subsection{Load application}

The loading of the single-stage ceramic tile grout seal with the indentor process is dynamic due to the timevarying load. However, the indentor's slow descent means that the loading process can be treated as a quasi-equilibrium. As such, simulation can be achieved as a static mode by incrementing the load and simultaneously manipulating the pressure distribution and contact interface. Simulation was subsequently carried out at six load levels: 100, 150, 200, 250 and $300 \mathrm{~N}$ and failure load. As the curvature of the vitrified enamel layer of the indentor face is minor, the contact area was assumed to be flat at each load level. Since many researchers $[\mathbf{1 2}, \mathbf{2 4}$, 25] have made the assumption that a rigid indentor will have frictionless contact in FE simulations of indentation testing, it was also assumed in this work that there was no frictional interaction between the indentor and the single-stage ceramic tile grout seal specimens. Through work with an FE model to simulate indentation testing, He and Veprek [26] pointed out that if the surface of the material was to be indentation-tested super-hard with an elastic modulus greater than $550 \mathrm{MPa}$, then the deformation of the indentor cannot be ignored. Since neither the surface nor the bulk of the single-stage ceramic tile grout seal is super-hard, the assumption of a rigid indentor is probable.

\subsection{Application of constraints}

It is assumed that the side and base of the surface of the single-stage ceramic tile grout seal is tightly adhered to the adjacent ceramic walls and grout respectively. Despite the indentation on to the single-stage ceramic tile grout seal, the nodes at these boundaries are 


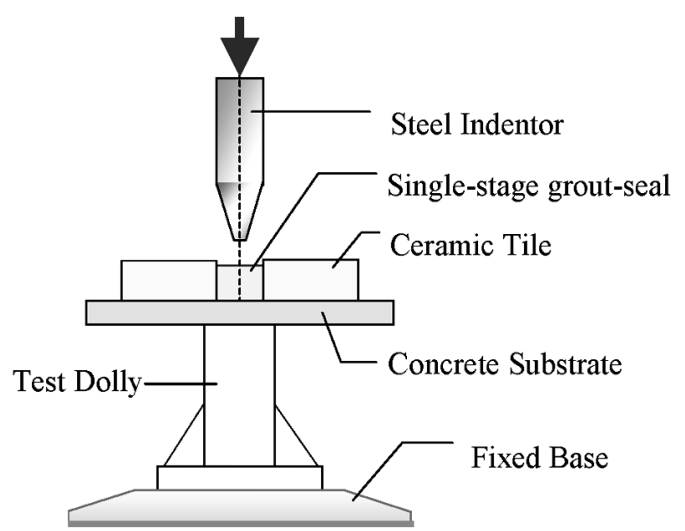

Fig. 3 Schematic diagram of the experimental set-up for the indentation tests

constrained in all directions. When the segment of the single-stage ceramic tile grout seal that is under the indentor face is being compressed, adjacent segments of unindented single-stage ceramic tile grout seal are likely to pose resistance to movement in the $y$ and $z$ directions at the common plane. Since the modelling of an adjoining grout seal would require much computational time, the model is simplified by assuming that the resistance in the $z$ direction at the common plane is infinite. This is conceivable as the adjoining sections of the singlestage ceramic tile grout seal will be infinitely long in comparison with the indented segment of the single-stage ceramic tile grout seal and can therefore be considered as a formidable barrier. Another unknown resistance in the $y$ direction is considered to be negligible, as indentation is likely to cause spreading of the single-stage ceramic tile grout seal in close proximity to the indentor only.

\section{COMPARISON OF THE FINITE ELEMENT MODEL RESULTS AND EXPERIMENTAL LOAD-DISPLACEMENT RESULTS}

It can be seen that Figs 4, 5 and 6 contrast the experimental and FE-generated load-displacement plots for geometries 1, 4 and 5 respectively (as listed in Table 1). The FE-generated load-displacement lines are plotted from the equations obtained by means of a regression analysis. Although the FE-generated model appears to be stiffer with a steeper gradient and higher elastic modulus, the results are in reasonable agreement with

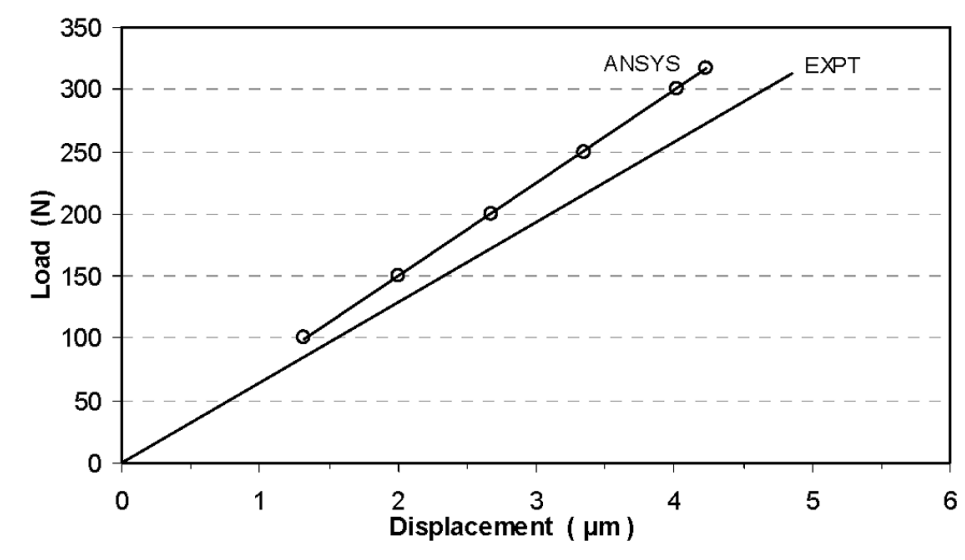

Fig. 4 Comparison of the load-displacement plot (geometry 1)

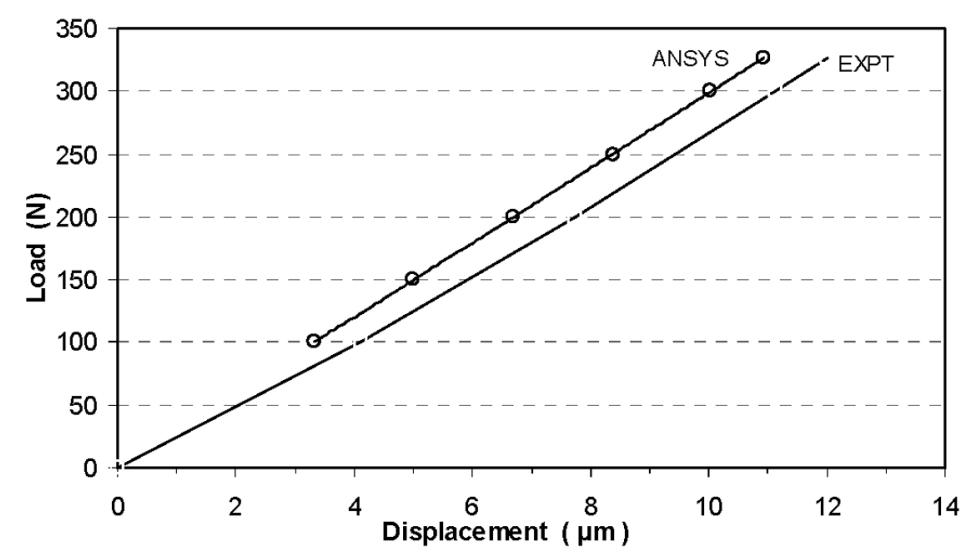

Fig. 5 Comparison of the load-displacement plot (geometry 4) 


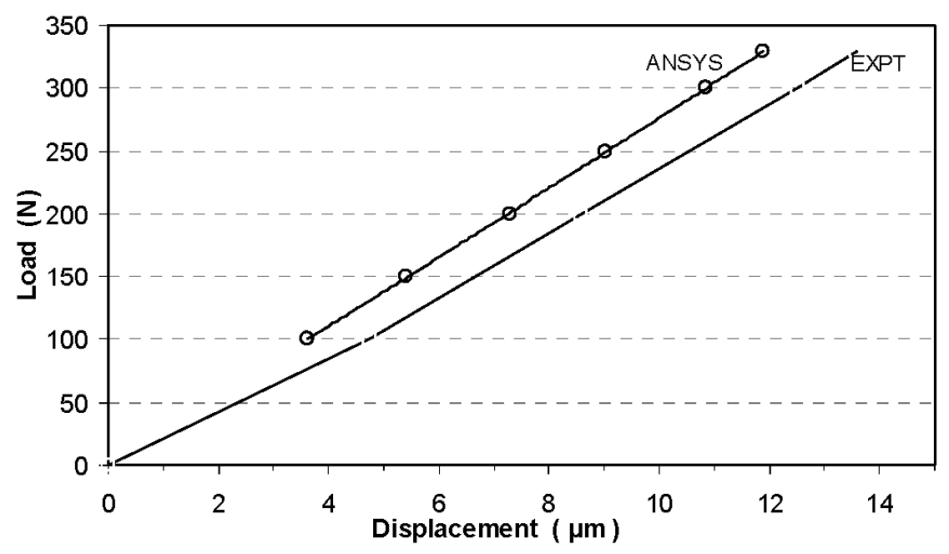

Fig. 6 Comparison of the load-displacement plot (geometry 5)

the actual experimental findings, with a percentage deviation of 13-17 per cent. Three implications can be drawn from this apparent agreement. Firstly, the assumption of the FE model to be a homogeneous and isotropic material with mechanical properties similar to glass is probable. Secondly, the constraints assumed and applied were appropriate. Finally, the dynamic loading can be successfully simulated by a static model through load increment with manipulation of the contact area and load distribution. In addition, all the plots suggest that the FE results are converging to the actual experiment results, with the percentage deviation decreasing as the load increases. One probable reason for this is that, at higher loads, the vitrified enamel layer on the surface of the single-stage ceramic tile grout seal would have failed, meaning that the bulk of the single-stage ceramic tile grout seal, which consists mainly of the crushed vitrified tile substrate, would be supporting the load. Because the elastic modulus and Poisson's ratio of this substrate are assumed to be similar to that of the glass, a convergence of results will occur.

\section{OPTIMIZATION OF THE CURVATURE OF THE VITRIFIED ENAMEL LAYER}

\subsection{Optimization objective}

When indented, the single-stage grout seal experiences two phases of failure. The first phase of failure is the functionality failure occurring at initial loadings, whereby cracks develop from the surface of the singlestage ceramic tile grout seal and propagate into the vitrified enamel layer. Although unsightly, such a failure is not disastrous as it could quite feasibly be repaired by means of HPDL treatment, always assuming that the damage is not too severe. However, as loading is further increased, a sufficient amount of yielding will take place, causing the entire single-stage ceramic tile grout seal to collapse under a catastrophic failure mode.
It was observed from the indentation experiments (see Table 3) that the load to failure increased as the curvature of the vitrified enamel layer increased. However, increasing curvature of the vitrified enamel layer will also bring about a higher stress concentration at the contact surface, thereby causing the vitrified enamel glaze to fail at a lower load capacity. From the experiments it was noted that the vitrified enamel glaze for the flat geometry failed at approximately $150 \mathrm{~N}$. The load-bearing capacity of the enamel glaze for the two curvilinear geometries lies below $50 \mathrm{~N}$.

The optimum curvature of the vitrified enamel layer must also consider the warning factor, whereby the failed enamel glaze would serve as an indication before catastrophic failure and allow time for maintenance. Thus, the objective of the curvature optimization must have two concerns: (a) the curvature of the vitrified enamel layer should provide the enamel glaze and the bulk of the single-stage ceramic tile grout seal with a reasonable load-bearing capacity and (b) simultaneously have sufficient indication of a catastrophic failure.

\subsection{Optimization approach}

Geometries of seven different curvatures of the vitrified enamel layer (as listed in Table 1) were modelled and simulated. Of the seven geometries tested, two surfaces were convex $(-0.2$ and $-0.7 \mathrm{~mm})$, one was flat $(0 \mathrm{~mm})$ and the remaining were concave $(+0.2,+0.4,+0.7$ and $+1.2 \mathrm{~mm}$ ). Since the indentation exhibits shear driven

Table 3 Experimental observations of the maximum Tresca stresses at the vitrified enamel layer during indentation

\begin{tabular}{lcll}
\hline Geometry & $\begin{array}{l}\text { Curvature } \\
(\mathrm{mm})\end{array}$ & $\begin{array}{l}\text { Enamel seal LBC } \\
(\mathrm{N})\end{array}$ & $\begin{array}{l}\text { Overall LBC } \\
(\mathrm{N})\end{array}$ \\
\hline 1 & 0 & 150 & 316.6 \\
4 & +0.7 & $<50$ & 326.2 \\
5 & +1.2 & $<50$ & 329.4 \\
\hline
\end{tabular}


yield, the material was idealized to be an elastic, perfectly plastic characteristic where the catastrophic failure and functionality failure of the single-stage ceramic tile grout seal would be related to the Tresca criterion, which states that failure occurs when the maximum difference of the principal stresses exceeds the yield strength. The yield strength is the compression yield strength of the vitrified tiles, which was $100 \mathrm{MPa}$. To predict the load-bearing capacity (LBC) of the vitrified enamel layer, the models were loaded at three load levels: 100, 200 and $300 \mathrm{~N}$. The maximum Tresca stresses at the enamel glaze contact surface were correlated with experimental observations. Finally, the criterion is applied to other untested geometries to predict their LBCs. The second phase of the analysis is to predict the overall LBC of the single-stage ceramic tile grout seal. Firstly, the loads to cause catastrophic failure on the three experimentally tested geometries were simulated with the FE model. Next, the Tresca stress contours were analysed and correlated with the experimental findings to implement a criterion to predict the overall LBC. Finally, the criterion is applied to untested geometries to predict the overall LBC.

\subsection{Observations from stress contours}

If geometries 3 to 5 are considered, whose contact areas and load distribution do not differ much, it can be seen that as the curvature of the vitrified enamel layer increases, the nominal stress decreases. This finding agrees with the theorem proved by Hsu [27], which states that when the position of both end points of the boundary curve to be varied are fixed, increasing the depth under the curve will ensure a decrease in stress. Table 4 shows that the Tresca stress contours at the three load levels indicate that the flat geometry experiences the least yielding on the contact surface. This is because the flat geometry has the maximum contact area with the indentor. As curvature of the vitrified enamel layer increases or decreases from a flat surface geometry, the contact area reduces. Moreover, it is noted that the reduction in contact area from the decrease of curvature of the vitrified enamel layer is more drastic and leads to a higher maximum Tresca stress, as can be seen from a comparison of geometry 6 and geometries 2 to 5 in Table 4 . Nevertheless, an agreement is seen in all the geometries that the maximum Tresca is located at the region of contact.

\subsection{Criterion for the prediction of load-bearing capacities}

From the Tresca stress contours at the three load levels of the experimentally tested geometries (1,4 and 5), it can be seen in Table 4 that the maximum Tresca stresses at the contact region have already exceeded the $100 \mathrm{MPa}$ yield strength of the vitrified enamel surface. This observation is in agreement with the research of Fisher-Cripps [28], which demonstrated that materials exhibit yield in indentation tests with spherical indentors, even at modest loads. It is clear that the enamel seal would fail only if the maximum Tresca stress exceeds the yield strength by a certain degree.

The ratio of maximum Tresca stress to yield strength is plotted against load in Fig. 7. Correlating with the experimental observations from Table 3, it was computed that for the vitrified enamel layer to fail, the maximum surface Tresca stress must be at least 3.5 times the yield strength. This criterion is applied to predict vitrified enamel layer LBCs for the untested geometries. From Fig. 7, geometries 6 and 7 with negative curvatures of the vitrified enamel layer will experience earliest failure of the enamel seal, at $10 \mathrm{~N}$. Geometries 2 to 5 , which have positive curvatures of the vitrified enamel layer, will experience failure of the glaze layer between 60 and $80 \mathrm{~N}$. The flat surface geometry, 1, has the highest enamel seal $\mathrm{LBC}$, at $150 \mathrm{~N}$.

From the Tresca stress distribution of the three experimentally tested single-stage ceramic tile grout seals at their failure loads given in Fig. 8, it can be seen that a significant portion of the cross-sectional area, approximately 29 per cent, is in the yield state for all three geometries. It is deduced that catastrophic failure occurs when 29 per cent of the single-stage ceramic tile grout seal is in the yield state. This criterion is applied to predict overall LBCs for untested geometries.

For geometries with negative curvatures of the vitrified enamel layer, the overhanging region experiences high tensile stresses during loading. As the single-stage ceramic tile grout seal is stronger in compression than tension, this overhanging region might collapse during the early loading phase. Since the model does not take

Table 4 Experimental results and observations in terms of load

\begin{tabular}{lccccccc}
\hline \multirow{6}{*}{ Load (N) } & $1(0)$ & $2(+0.2)$ & $3(+0.4)$ & $4(+0.7)$ & $5(+1.2)$ & $6(-0.2)$ & $7(-0.7)$ \\
\cline { 2 - 7 } & 236 & 404 & 721 & 804 & 898 & 2510 & 2640 \\
100 & 440 & 804 & 1420 & 1650 & 1800 & 4590 & 5030 \\
300 & 668 & 1230 & 2110 & 2490 & 2690 & 6660 & 7810 \\
\hline
\end{tabular}




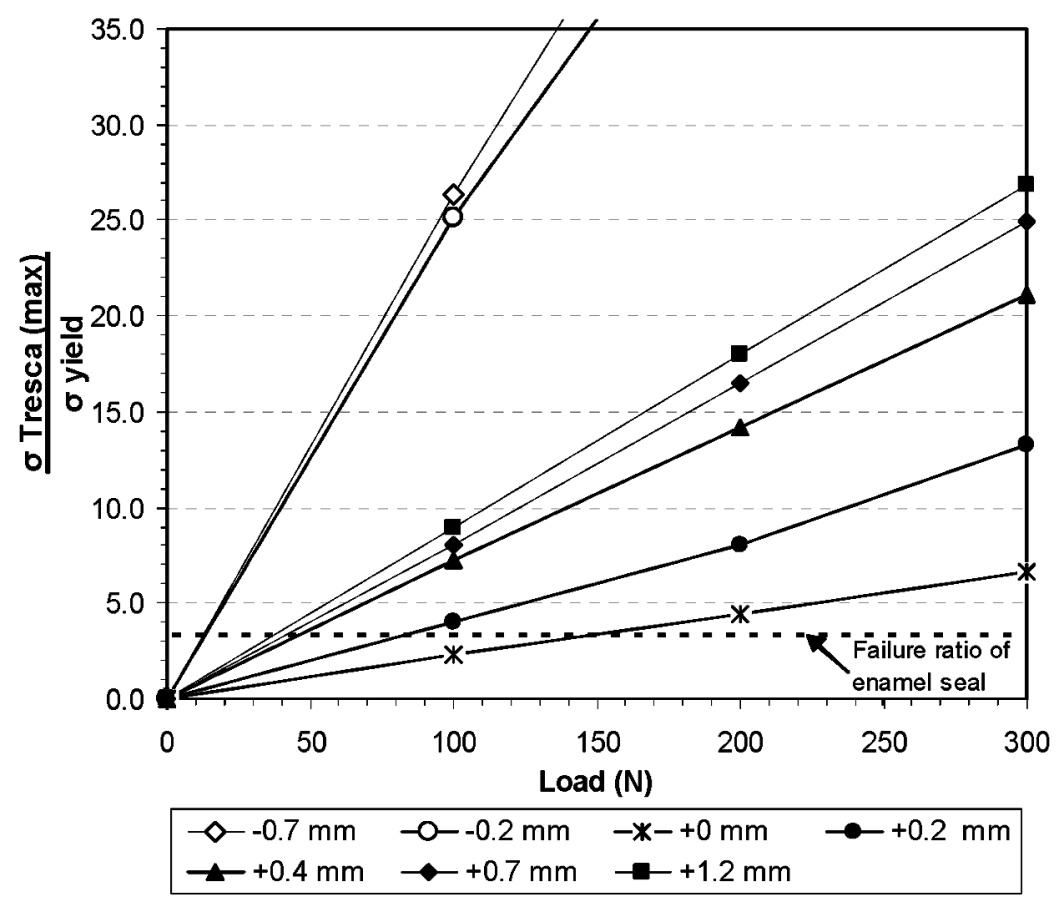

Fig. 7 Plot of the stress ratio against load for various radii of curvature

this into account, it is likely that the overall LBCs for negative curvatures of the vitrified enamel layer are lower than the predicted values. From the overall LBCs versus the curvature of the vitrified enamel layer in Fig. 9, it is seen that the overall LBC increases with curvatures of the vitrified enamel layer. The reason is that increased curvature of the vitrified enamel layer would generate a larger volume of single-stage ceramic tile grout seals, so that for 29 per cent of the area to reach the yield state would require higher loads. However, this trend is not perpetual as the positive gradient is decreasing. The plot indicates that the peak might

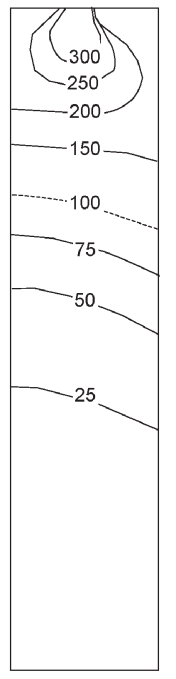

$\# 1 *$ $316.6 \mathrm{~N}$

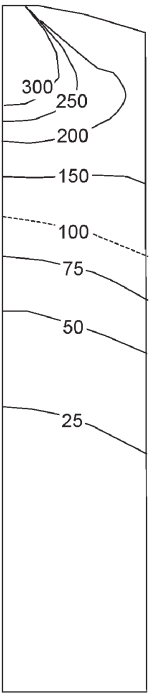

\#2 $320.0 \mathrm{~N}$

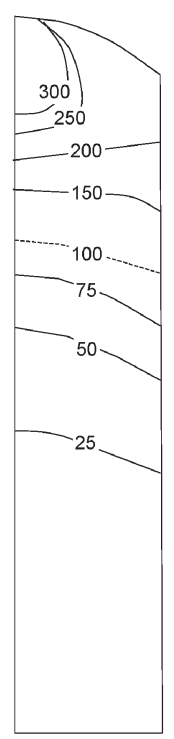

\#3 $323.0 \mathrm{~N}$

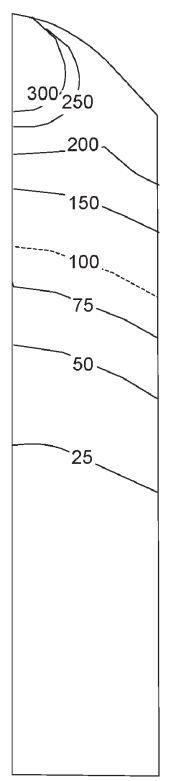

\#4* $326.2 \mathrm{~N}$

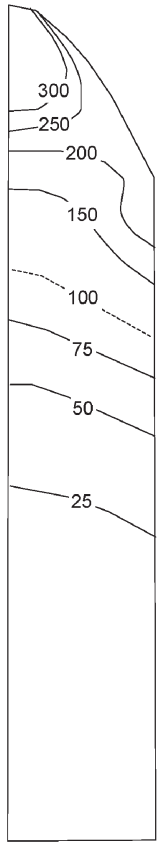

\#5*

$329.4 \mathrm{~N}$

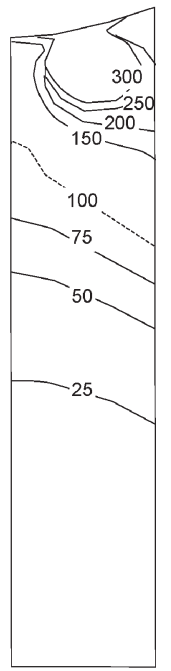

\#6

$312.0 \mathrm{~N}$

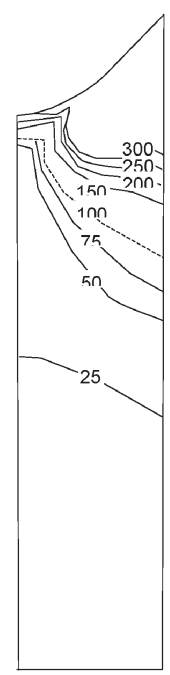

\#7

$305.0 \mathrm{~N}$

Fig. 8 Stress contours at failure loads (units are N) 


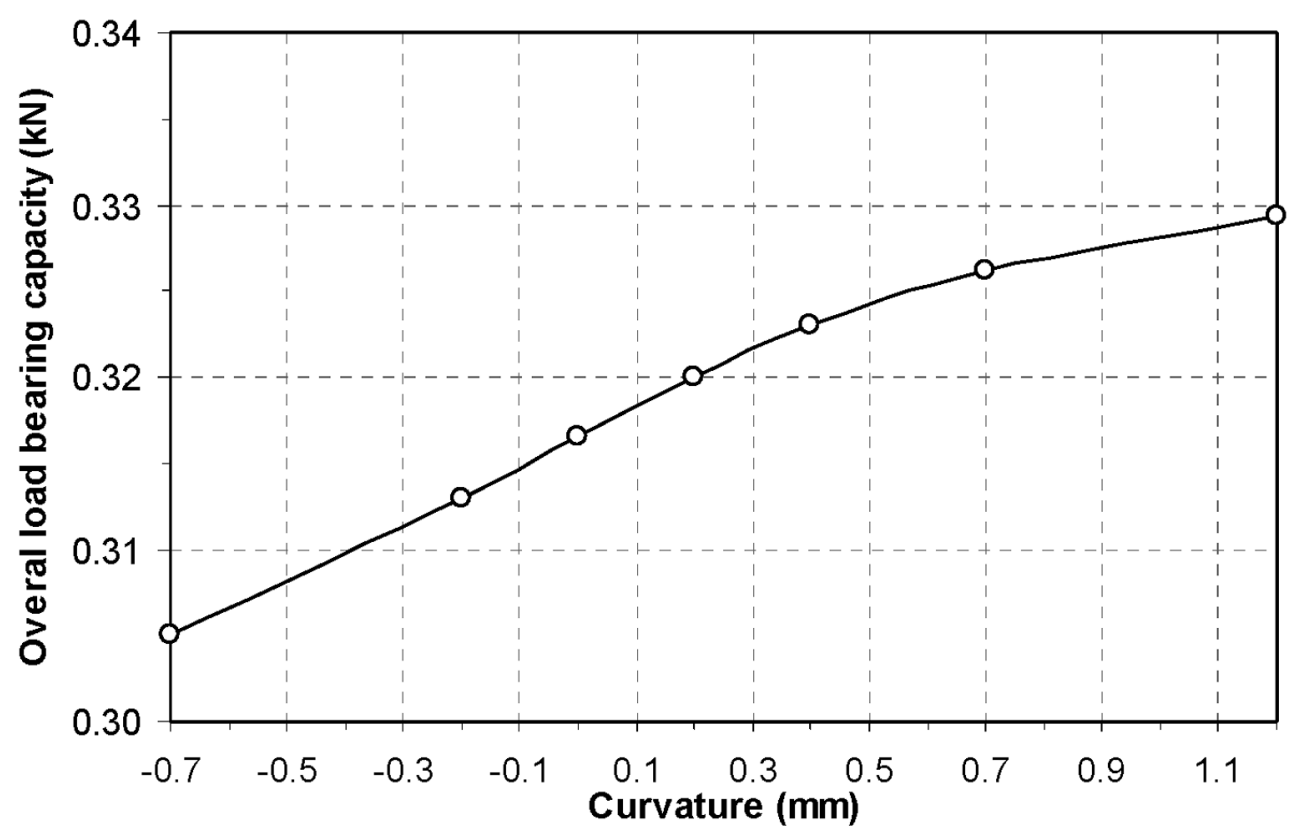

Fig. 9 Trend of the overall load-bearing capacity with different curvatures of the vitrified enamel layer

occur somewhere near a curvature of the vitrified enamel layer of $1.2 \mathrm{~mm}$.

\subsection{Optimum design}

European standards [29] specify cementitious grout to have a compressive strength of $45 \mathrm{~N} / \mathrm{mm}^{2}$. In Fig. 10, the two LBCs and their differences are plotted against curvatures of the vitrified enamel layer. Generally, a large LBC difference gives an early indication that the single-stage ceramic tile grout seal is damaged and requires repair. As all the values are greater than $150 \mathrm{~N}$ (three times higher than the standard specification of the resin grout), it is concluded that all geometries would give a sufficient reaction time. In addition, it is noted that the overall LBCs for all the geometries are above $300 \mathrm{~N}$, which is seven times higher than the standard specification. The deciding factor lies in the vitrified enamel layer. For curvatures of the vitrified enamel layer

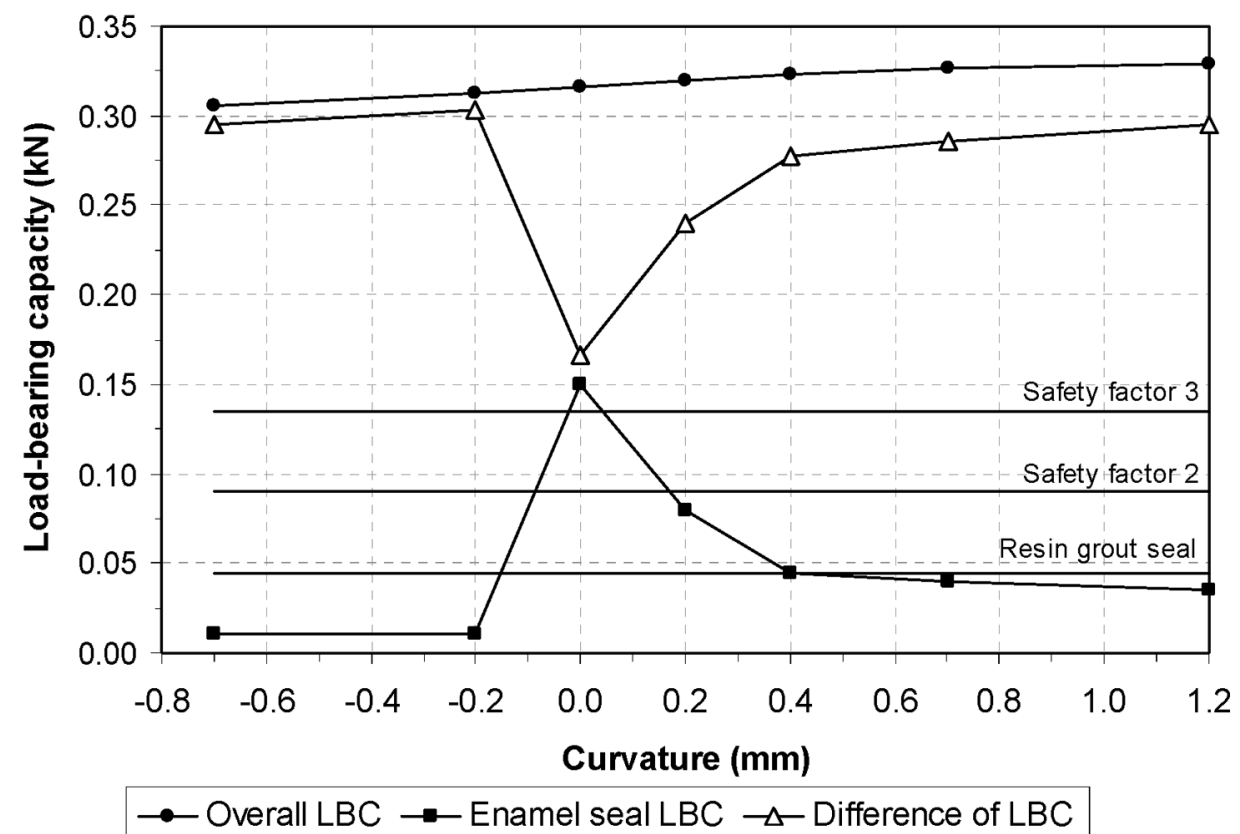

Fig. 10 Load-bearing capacities with curvature of the vitrified enamel layer 
less than $-0.2 \mathrm{~mm}$ and larger than $+0.4 \mathrm{~mm}$, a large LBC difference would result in an overly weakened vitrified enamel layer that would require frequent repairs, since the increased sensitivity is not accompanied by any significant improvement in the overall LBC. To satisfy the standard European specification, a curvature of the vitrified enamel layer of -0.15 to $+0.40 \mathrm{~mm}$ would be sufficient. However, for a safety factor of two, the curvature of the vitrified enamel layer ought to be within -0.08 to $+0.15 \mathrm{~mm}$. Subsequently, for a safety factor of three, the curvature of the vitrified enamel layer should be kept between $-0.02 \mathrm{~mm}$ and $+0.05 \mathrm{~mm}$. The optimum design is a flat surface geometry.

\section{CONCLUSIONS}

From the reasonable agreement of 13-17 per cent of the load-displacement results obtained with the finite element (FE) model and the actual experimental results, it is concluded that the FE model is capable of simulating the indentation testing. The constraints and loading were appropriately assumed and applied. Having said this, the idealization of the graded substrate to be a homogeneous and isotropic model with constant elastic modulus and Poisson's ratio similar to glass is the main cause of minor discrepancies and is the reason that the FE model appears to be stiffer with a steeper gradient and higher elastic modulus. The work revealed that the stress distribution derived from the FE model, along with the Tresca failure criterion, was capable of predicting load bearing capacities (LBCs) of the single-stage ceramic tile grout seal. The vitrified enamel surface layer was found to fail when the maximum Tresca stress is 3.5 times the yield strength and the entire single-stage ceramic tile grout seal collapsed when 29 per cent of its cross-section is above the yield state. From the investigation it was noticed that the slight improvements in the overall LBC for increased curvatures of the vitrified enamel layer were offset by a greater degradation of the LBC of an overly weakened vitrified enamel surface layer as a result of a higher built-up in stress concentration at the apex. However, the LBC differences of all investigated geometries of the vitrified enamel surface layer would allow a reasonable reaction time, as they are three times higher than the standard European specification. In addition, all the geometries have satisfactory overall LBCs, seven times higher than the standard requirement. From the FE model, it was found that the optimum design with the highest safety factor was the flat surface geometry. Having said this, depending upon the environment of operation, the design can be manipulated depending on the required safety factor. For instance, with a safety factor of one, the curvature of the vitrified enamel layer should fall within the region of -0.15 to $0.40 \mathrm{~mm}$; for a safety factor of two, the curvature of the vitrified enamel layer should be within -0.08 to $+0.15 \mathrm{~mm}$; and for a safety factor of three, the curvature of the vitrified enamel layer should lie between $-0.02 \mathrm{~mm}$ and $+0.05 \mathrm{~mm}$.

\section{REFERENCES}

1 Taylor, G. D. Construction Materials, 1991 (Longman Scientific and Technical, London).

2 Lawrence, J., Li, L. and Spencer, J. T. A two-stage ceramic tile grout sealing process using a high power diode laser I. Grout development and materials characteristics. Opt. Laser Technol., 1998, 30, 205-214.

3 Lawrence, J., Li, L. and Spencer, J. T. A two-stage ceramic tile grout sealing process using a high power diode laser II. Mechanical, chemical and physical properties. Opt. Laser Technol., 1998, 30, 215-223.

4 Lawrence, J. and Li, L. Finite element analysis of temperature distribution using ABAQUS for a laser-based tile grout sealing process. Proc. Insn. Mech. Engrs, Part B: J. Engineering Manufacture, 2000, 214(B6), 451-461.

5 Lawrence, J., Schmidt, M. J. J., Li, L., Edwards, R. E. and Gale, A. G. A portable high-power diode laser-based singlestage ceramic tile grout sealing system. Opt. Laser Technol., 2002, 34, 27-36.

6 Lawrence, J., Li, L., Edwards, R. E. and Gale, A. G. A comparative investigation of the wear characteristics of a high power diode laser generated single-stage and commercial epoxy tile grout. Wear, 2002, 252, 88-95.

7 Zhang, Y. M., Kovacevic, R. and Li, L. Characterisation and real time measurement of geometrical appearance of the weld pool. Int. J. Mach. Tools Mf., 1996, 36, 799-816.

8 Bull, C. E., Stacey, K. A. and Calcraft, R. On line weld monitoring using ultrasonic. J. Non-Destructive Test., 1993, 35, 57-64.

9 Teng, T. L., Fung, C. P. and Chang, P. H. Effect of weld geometry and residual stresses on fatigue in butt-welded joints. Int. J. Pressure Vessels Piping, 2001, 79, 467-482.

10 Karkhin, V. A. Effects of weld shape on the distribution of stresses, in tension, in butt joints in thick metal. Avt. Svarka, 1985, 9, 25-28.

11 Shuzalec, A. Shape optimisation of weld surface. Int. J. Solid Structs, 1989, 25, 23-31.

12 Dong, X. D. and Darvell, B. W. Stress distribution and failure mode of dental ceramic structures under Hertzian indentation. Dental Mater., 2003, 19, 145-153.

13 ASTM C597-591 Standards Test methods for Compressive Strength of Chemical-Resistant Mortars, Grouts, Monolithic Surfaces and Polymer Concrete (American Society for Testing and Materials, West Conshohocken, Pennsylvania).

14 Pedersen, P. and Lauren, C. L. Design for minimum stress concentration by finite elements and linear programming. Struct. Meth., 1983, 10, 375-391.

15 Kristensen, E. S. and Madsen, N. F. On the optimum shape in fillets in plates subjected to multiple in-plane loading cases. Int. J. Numer. Meth. Engng, 1985, 10, 1007-1009.

16 Bhavikatti, S. S. and Ramakrishnan, C. V. Optimum shape design of rotating disks. Computer Structs, 1980, 11, 397401. 
17 Braibant, V. and Fleury, C. Shape optimal design using Bspline. Comput. Meth. Appl. Mech. Engng, 1984, 44, 247-267.

18 Yang, R. J. and Choi, K. K. Accuracy of finite element based shape sensitivity analysis. Struct. Mechanics, 1985, 13, 223-289.

19 Sandgren, E. and Wu, S. J. Shape optimisation using the boundary element method with substructuring. Numer. Meth. Engng, 1988, 25, 1913-1924.

20 Hsu, L. L., Sheppard, S. D. and Wildle, D. J. The curvature function method for two-dimensional shape optimisation under stress constraints. Computer Structs, 1993, 55, 647-657.

21 Fisher-Cripps, A. C. Analysis of instrumented indentation test data for functionally graded materials. Surf. Coating Technol., 2003, 168,136-141.

22 Azhdari, A., Obata, M. and Sia N. N. Alternative solution methods for crack problems in plane anisotropic elasticity, with examples. Int. J. Solid Structs, 2000, 37, 6433-6478.

23 Degarmo, E. P., Black, J. T. and Kohser, R. A. Materials and Processes in Manufacturing, 1997 (Prentice-Hall, Upper Saddle River, New Jersey).
24 Lin, S., Hills, D. A. and Warren, P. D. A theoretical investigation of sharp indentation testing of a nearly-brittle material, with some experimental results. J. Mech. Phys. Solids, 2000, 48, 2057-2075.

25 Eriksson, C. L., Larsson, P. L. and Rowcliffe, D. J. Strain hardening and residual stress effects in plastic zones around indentations. Mater. Sci. Engng. A, 2003, 340, 193-203.

26 He, J. L. and Veprek, S. Finite element modelling of indentation into super-hard coatings. Surf. Coating Technol., 2003, 163-164, 374-379.

27 Hsu, Y. L. Zero order optimization methods for twodimensional shape optimization. PhD thesis, Stanford University, California, 1992.

28 Fisher-Cripps, A. C. Elastic-plastic behaviour in materials loaded with a spherical indentor. J. Mater. Sci., 1997, 32, 727-736.

29 European (EN) Standard Performance Requirements for Grouts, 1997. 
Copyright of Proceedings of the Institution of Mechanical Engineers -- Part B -Engineering Manufacture is the property of Professional Engineering Publishing and its content may not be copied or emailed to multiple sites or posted to a listserv without the copyright holder's express written permission. However, users may print, download, or email articles for individual use. 